\title{
Biocompatible surgical meshes based on decellularized human amniotic membrane
}

\author{
Peina Shi ${ }^{1}$, Mengna Gao ${ }^{1}$, Qiuxia Shen ${ }^{1}$, Lei Hou ${ }^{1}$, Yabin Zhu ${ }^{1}$, Jun Wang ${ }^{2}$ \\ ${ }^{1}$ The Medical School, Ningbo University, Ningbo, China, 315211 \\ ${ }^{2}$ Obstetrics and Gynecology Department of the Sixth Hospital, Shanghai Jiaotong University,
}

Shanghai, 201306

\author{
*Corresponding author, E-mail: zhuyabin@nbu.edu.cn \\ Tel: +86-0574-87609592; \\ Fax: +86-0574-87608638
}

\begin{abstract}
Meshes play important roles to repair human tissue defect. In this work, human amniotic membrane (HAM) was decellularized and explored the efficacy as an implantable biological mesh. Surfactant, hypertonic saline, lipase and DNAase were used individually or collectively to remove all cell components and remain the extracellular matrix. Results of H\&E and DAPI staining demonstrated that the method of surfactant and lipase combining with DNAase is the most effective treatment for HAM decellularization. Primary smooth muscle cells were seeded to evaluate the decellularized HAM's (dHAM) in vitro cytocompatibility. The in vivo test was performed via implantation at rabbits' uterus with clinic polypropylene mesh (PP) as the control. The results indicated that dHAM possessed good biocompatibility and will be a potential candidate for biological mesh.
\end{abstract}

Keywords: Biological mesh; Human amniotic membrane; Decellularization, Biocompatibility.

\section{Introduction}

With the development of technology in tissue engineering, biological meshes play more and more crucial roles in repairing of abdominal wall hernia, vaginal, uterus and bladder prolapse, pelvic floor defects, and interventional devices like vascular/endovascular grafts, biological substitutions for resected tissues [1-5]. Most traditional surgical meshes were made from artificial materials or animal tissues to provide permanent reinforcement or temporary substitutes. For 
example, polypropylene mesh (PP) is used for urological and urogynecological surgery and polyester mesh (PE) used for hernia repair [6,7]. However, these implants have been associated with inflammation, infection, tumor or even carcinogenicity due to the material's hydrophobicity and consequently poor tissue integration [8-10]. Researchers start to study on improving meshes' chemistry via using plant-/animal-derived materials or surface crosslinking with hydrophilic components to enhance the meshes' wettability $[11,12]$.

Human amniotic membrane (HAM) is a translucent thin membrane derived from the most inner layer of placenta, with no blood vessels, nerves and lymph vessels. It possesses properties like low-inflammation, low-immunogenicity and inhibition of leukocyte infiltration, and thus has been explored for a variety of clinical applications like grafts for eye injuries and dressing for integumentary wounds [13-15]. The basic structure of HAM consists of three layers: epithelial, basement layer and avascular stromal layer. In order to diminish the immunogenicity originated from cells and improve the in vivo biocompatibility, people always separated its basement layer (containing epithelial cells) and conduct decellularization performance to obtain a denuded human amniotic scaffold with $\sim 50 \mu \mathrm{m}$ thickness [16]. Some attempts have been made to decellularize HAM [17]. For example, surfactant like sodium dodecyl sulfate (SDS), ethylenediaminetetraacetate dehydrate (EDTA), sodium hydrochloride or mechanical scraping were used $[18,19]$. However, these treatments cannot remove amniotic epithelial cells completely and shall be optimized the detail procedures.

In this work, we focused on decellularization of HAM and in vitro/in vivo testing, aiming at producing a biocompatible mesh. In order to settle the HAM's problems like over-softness and weak mechanical properties, Poly (ester urethane) (PU) was selected as physical support because of PU's non-toxicity and biodegradability with good mechanical properties like strength, elasticity and stability, which was much proved in our previous studies or other's work [20-24]. Decellularized HAM (dHAM) was expected to provide biocompatibility while PU provided physical strength. The dHAM/PU/dHAM hamburg composite (abbreviated as dHAM/PU) was therefore fabricated. It was supposed to possess both anti-inflammation and good mechanical strength. This kind of HAM-based scaffold was not reported in literatures. In vitro and in vivo 
evaluations were performed on $\mathrm{dHAM} / \mathrm{PU}$ membrane. The results demonstrated the enhanced interaction with smooth muscle cell and high integration with rabbit abdominal tissue. This dHAM/PU was anticipated to serve as an implantable biological mesh in future.

\section{Experimental section}

\subsection{Materials}

Poly(ester urethane) (PU, 58213 NAT 022) was purchased from Estane Co., China. Triton X-100 (Solarbio, China), Antibiotics Solution (Penicillin and streptomycin, Beyotime Institute of Biotechnology, China), DNAase (Sinopharm Chemical Reagent Co., China), lipase (Solarbio, China), Dulbecco's modified Eagle's medium (DMEM, Hyclone, USA), fetal bovine serum (FBS, TransGen Biotech, China), Methylthiazolyldiphenyl-tetrazolium bromide (MTT, BioDee, China) were used as obtained.

Fresh HAM was collected immediately after delivery from the Affiliated Hospital of Ningbo University, with prior consent of the pregnant women. All HAMs used in experiments were obtained from healthy pregnant.

\subsection{Decellularization of HAM}

The fresh HAM was separated from placenta, rinsed away all blood and other dirties in phosphate buffered saline(PBS), rapidly sterilized in $75 \%$ alcohol/water for $10 \mathrm{~s}$, and washed away the alcohol with PBS. It was then put in PBS containing antibiotics $(200 \mathrm{U} / \mathrm{mL})$ under shaking for $1 \mathrm{~d}$ with changing the PBS for each $12 \mathrm{~h}$.

These pretreated HAMs were divided into 4 groups for decellularization performance; (1) Surfactant + DNAase (SD): HAM was immersed in 1\% Triton X-100 for 1, 2 or 3d, respectively, followed by treatment with DNAase/PBS solution(2000U/L) for $3 \mathrm{~h}$ at $37^{\circ} \mathrm{C}$; (2) Hypertonic saline + DNAase (HD): HAM was incubated in $1 \mathrm{M}, 2 \mathrm{M}$ or $3 \mathrm{M} \mathrm{NaCl} / \mathrm{PBS}$ solution separately for 1,2 or 3d, followed by DNAase treatment as above; (3) Lipase + DNAase (LD): HAM was firstly incubated in lipase/PBS solution (concentration of 100, 200, 500, 800, 1000,1200 1500, 1800, 2000 and $2500 \mathrm{U} / \mathrm{L}$ was explored individually to select a favorable solution) for $10 \mathrm{~h}$ at $37^{\circ} \mathrm{C}$ It was then treated in DNAase solution as above. (4) Surfactant + Lipase + DNAase (SLD): HAM 
was firstly treated in $1 \%$ Triton $\mathrm{X}-100$ for $14 \mathrm{~h}$ (changed the solution at $7 \mathrm{~h}$ ), followed by incubation in $2000 \mathrm{U} / \mathrm{L}$ lipase solution for $10 \mathrm{~h}$ at $37{ }^{\circ} \mathrm{C}$. Finally, it was incubated in $2000 \mathrm{U} / \mathrm{L}$ DNAase/PBS for $3 \mathrm{~h}$ at $37^{\circ} \mathrm{C}$ PBS rinsing (for $24 \mathrm{~h}$ with changing PBS at $12 \mathrm{~h}$ ) was performed in each procedure. All PBS was supplemented with $200 \mathrm{U} / \mathrm{mL}$ antibiotics.

All samples after decellularization were fixed in $4 \%$ paraformaldehyde/PBS for the following characterizations.

\subsection{HAM characterizations}

These fixed samples were rinsed by water completely, dehydrated with a graded series of ethanol, embedded in paraffin wax on Paraffin Embedding machine (EC350, Thermo Scientific,USA), and sectioned at $5.0 \mu \mathrm{m}$ on Microtome (HM325, Thermo Scientific, USA).Then the slices were mounted on glass, dewaxed by dimethylbenzene, rehydrated, and stained with hematoxylin for nucleic acid and eosin for cytoplasm. They were observed on Inverted Phase-contrast Microscope (FV1000, Olympus, Japan) to monitor the decellularizing effectiveness. The clean HAM was examined as the control reference.

These decellularized HAM (dHAM) was stained by 4',6-diamidino-2-phenylindole (DAPI, Sigma) to expose the existed nuclear matters. dHAM was ruptured in $0.5 \%$ Triton X-100 for 20 min,, and then dipped in DAPI/PBS $(5 \mu \mathrm{g} / \mathrm{mL})$ for $10 \mathrm{~min}$. The samples were examined under Fluorescence Inverse Microscope (FV2000, Olympus, Japan). The DNA content in cell nucleus will display as blue fluorescence.

The HAM morphology was examined on Scanning Electron Microscope (SEM, Hitachi S3400, Japan) after samples were surface metalized by gold on Ion Sputter (E-1010, Hitachi, Japan).

\subsection{Fabrication of dHAM/PU composite mesh}

PU was dissolved in 1,4-dioxane to get the concentration of $0.125 \mathrm{~g} / \mathrm{mL}$. This PU solution was cast on smooth glass to obtain a membrane with $\sim 0.3 \mathrm{~mm}$ thickness. The wet dHAM (treated with SLD method, i.e. $1 \%$ Triton X-100 for $1 \mathrm{~d}, 2000 \mathrm{U} / \mathrm{L}$ lipase for $10 \mathrm{~h}$ and 2000U/L DNAase for $3 \mathrm{~h}$ ) was coated evenly on PU membrane and dried in oven for $10 \mathrm{~h}$ at $37{ }^{\circ} \mathrm{C}$. The coating was performed repeatedly on the other side to get the dHAM/PU/dHAM hamburg composite 
(abbreviated as dHAM/PU).

\subsection{In vitro cytocompatibility evaluation}

Primary smooth muscle cells (SMCs) were digested and proliferated from rabbit esophagus [20]. Membranes of dHAM/PU and PU were punched into a disc with the diameter of $6.4 \mathrm{~mm}$. These round membrane were sterilized in $75 \%$ ethanol for $4 \mathrm{~h}$ and then rinsed ethanol away with sterile PBS. SMC ( $3^{\sim} 5^{\text {th }}$ passages) were seeded on membranes at the density of $4 \times 10^{4} / \mathrm{mL}, 200 \mu \mathrm{L}$ per well in 96-well plate (Corning Co., USA). SMCs were cultured in DMEM containing 10\% FBS and $100 \mathrm{U} / \mathrm{mL}$ antibiotics in a $\mathrm{CO}_{2}$ incubator (HF90, Heal Force, China) with 5\% humidity at $37^{\circ} \mathrm{C}$. The culture medium was changed every $2 \mathrm{~d}$.

To observe the cell morphology under optical microscopy, the cultures were stained with hematoxylin and eosin dye because of the poor transparency of the composite membrane. Cells were rinsed with PBS and fixed in $4 \%$ paraformaldehyde for $4 \mathrm{~h}$, rinsed by PBS to completely remove the residual paraformaldehyde, and immersed in $0.5 \%$ triton-X100/PBS for $15 \mathrm{~min}$ and rinsed with water. Cells were stained with hematoxylin for $30 \mathrm{~min}$, rinsed in $1 \%$ hydrochloric acid/alcohol for $10 \mathrm{~s}$ to remove the excess dye. Subsequently, cells were stained in eosin for $1 \mathrm{~min}$.

For mitochondrial activity assay, cells were seeded on dHAM/PU and PU membrane (used as control), and kept in 96-well culture plate with tissue culture polystyrene plate (TCPS) as the positive reference. After 1, 3, 7 or 10d, respectively, the cell-seeded membranes were transferred into a new plate. MTT/PBS solution ( $20 \mu \mathrm{L}, 5 \mathrm{mg} / \mathrm{mL}$ ) was added to each sample well (in 96-well plate) and incubated at $37^{\circ} \mathrm{C}$ for $4 \mathrm{~h}$, followed by addition of $150 \mu \mathrm{L}$ dimethyl sulfoxide (DMSO) to dissolve the purple formazan crystal. The absorbance at the wavelength of $490 \mathrm{~nm}$ (OD) was determined on an ELISA colorimeter (MaxM5, Labsystems Spectra, USA). The absorbance of the same solvent in an unseeded well (without formazan crystal) was used as blank reference. OD values were averaged from 5 repeats for each sample type.

\subsection{Immunohistochemistry staining}

Immunohistochemistry staining was performed on the cultures using anti- $\alpha$-Smooth Muscle Actin (SMA, Boster, China) as the primary antibody. A standard immunostaining protocol as manual introduced was used. Samples were washed with PBS and fixed in 4\% paraformaldehyde 
for $30 \mathrm{~min}$. They were first incubated in $0.1 \%$ Triton X-100 for 10 min, rinsed triply with PBS for 10 min each time, and blocked in $4 \%$ goat serum for $1 \mathrm{~h}$ to minimize non-specific binding. Cells were then incubated in mouse anti- $\alpha$-SMA (dilution 1:200 in PBS) for $1 \mathrm{~h}$ at $37{ }^{\circ} \mathrm{C}$. After rinsing with PBS triply, the samples were incubated in goat anti-mouse $\operatorname{IgG}(\mathrm{H}+\mathrm{L})$ secondary antibody conjugated with FITC (1:50 dilution in PBS, Boster) for $1 \mathrm{~h}$. Rinsing with PBS triply, samples were dipped in DAPI/PBS ( $3 \mu \mathrm{g} / \mathrm{mL}$ ) for 5 min to reveal the nuclei. In the stained image, SMC's actin displayed green fluorescence and nucleus displayed blue.

\subsection{In vivo testing}

In order to enhance the tissue integration, dHAM/PU membrane was manually punched multi-holes with stainless steel needle (14G, inner/external diameter, $1.45 / 1.8 \mathrm{~mm}$, ratio of pore/membrane, 1:1, Figure 9 a0, inserted). 2 adult rabbits ( 1 year-old, $4-6 \mathrm{~kg}$ ) were used in this experiment. The rabbits were anesthetized with 3\% pentobarbital sodium (intravenous injection, $12 \mathrm{mg} / \mathrm{kg})$. dHAM/PU composite mesh $(1 \times 1 \mathrm{~cm})$ was implanted at uterus through rabbit's abdomen (Figure 9 a0, black arrow). Polypropylene mesh (PP, Marlex) supplied by Affiliated Hospital was used as the control (Figure 9 a0, red arrow). After 2 or 10 months, dHAM/PU and PP mesh was explanted with small amount of tissue, fixed by $4 \%$ paraformaldehyde, sectioned at 6 $\mu \mathrm{m}$, and performed $\mathrm{H} \& \mathrm{E}$ staining.

The animals used in this study were treated as required by the Ethical Committee of Ningbo University and in accordance with NIH's Principles of Laboratory Animal Care.

\subsection{Statistical Analysis}

Statistical analyses were performed with ANOVA software to evaluate the differences between groups. $\quad p$ values less than 0.05 were considered to be significant. Data were presented as mean \pm standard deviation $(\mathrm{SD})$.

\section{Results and discussions}

\subsection{Decellularization and the topography of HAM}

Methods like SD, HSD, LD and SLD with variety of solution concentrations, processing intervals and temperatures were explored to establish an effective approach to peel off all 
epithelial cells but maintain the ECM integrity. Results of HE and DAPI staining for cell cytoplasm and nucleus show that HAM consists of epithelium and mesenchymal connective tissue (Figure $1 \mathrm{a} 0$, arrow and triangle), which is the amnion histology as reported [25]. Cuboidal epithelial cells live compactly on basement membrane to line the HAM topology (Figure $1 \mathrm{a} 0$, inserted). Blue fluorescence in Figure 2 a0 displayed the orderly-arranged epithelial nuclei. These epithelial cells were decellularized using 4 methods separately, i.e. SD, HD, LD and SLD (the detailed procedures were described in "Experimental Section"). The processing parameters were compared among reagent selection, concentration of solutions, treatment time and temperatures. For the method of SD, HD or LD, the results of both HE and DAPI staining showed cells were not entirely removed; some nuclei or debris exposed (Figure 1, a1-a3 and Figure 2, a1-a3). Comparatively, the fewest nuclei or fragments left after HAM was treated by LD method, i.e. lipase/PBS (2000u/L) for $10 \mathrm{~h}$ and DNAase (2000U/L) for $3 \mathrm{~h}$ at $37^{\circ} \mathrm{C}$ (Figure 1 a3 and Figure 2 a3). As literature [26] reported, Triton X-100 is a kind of non-ionic surfactant and able to denaturalize protein gently. It was found that Triton X-100 destroyed the normal structure of the amniotic epithelial cell membrane to let DNAase infuse in and enzymolyzed the nuclear apparatus. Lipase, an enzyme able to catalyze the hydrolysis of lipids, was used to digest the lipids of epithelial cells. But it is not sufficient enough to enzyme all lipids by itself [27]. Thus, some nuclei or DNA debris were still existed in the treated HAM. Moreover, the extracellular matrix (ECM) was over-loosened, particularly under the rigorous treatment parameters like longer treatment time ( 2 or $3 \mathrm{~d}$ for Triton X-100) and greater concentration of solution (2500 U/L for lipase). Excessive enzyme reaction in method SD, HD or LD must have occurred not only on cell components but also on the ECM, resulting in the destructive mechanics of HAM.

Hopefully, we modified the methods, combined Triton X-100 and lipase to denature the cell membrane, and then used DNAase to digest all nuclear matters. Expectedly, epithelial cells were removed effectively and completely after treatment with method SLD, i.e. $1 \%$ Triton X-100 for 1 d, $2000 \mathrm{U} / \mathrm{L}$ lipase for $10 \mathrm{~h}$ and 2000U/L DNAase for $3 \mathrm{~h}$, as shown in Figure 3; seldom nucleus exhibited and ECM appears integration from HE and DAPI staining. Here, Triton X-100 combined with lipase ruined the epithelial cell's membrane. Thus, DNAase can permeate into 
nuclei membrane to enzymolyze the DNA content.

SEM examination revealed the topographic alteration (Figure 4). The apparent ECM fibers exhibited on dHAM (Figure 4b) but plumpy and cobblestoned topology on the original HAM (Figure 4a). Therefore, we treated HAM with method SLD (1\% Triton X-100 for $1 \mathrm{~d}, 2000 \mathrm{U} / \mathrm{L}$ lipase for $10 \mathrm{~h}$ and 2000U/L DNAase for $3 \mathrm{~h}$ ) to obtain the decellularized HAM (dHAM). It was then combined with PU membrane to prepare dHAM/PU/dHAM hamburg composite mesh. After H\&E staining (Figure 5 a), the pink dHAM was covered tightly at both sides of PU membrane. It seems there was no interspace between PU and dHAM (Figure 5 a, arrow in the inserted picture). However, under higher resolution (SEM observation), there was still a small gap in the interface (Figure $5 \mathrm{~b}$, arrow in the inserted picture). Moreover, PU membrane surface was not very smooth. There existed some micro-pores originated from solvent evaporation during PU membrane fabrication using solution casting method. It had been observed in our previous experiment [28]. We thus inferred that $\mathrm{dHAM}$ and PU was connected via some physical interaction due to the different surface energy of these two materials. This kind of interaction was considered to be fragile. We are now digging for more effective method to connect HAM and PU.

\subsection{Evaluation of in vitro cytocompatibility}

The cytocompatibility of dHAM/PU composite was evaluated via primary smooth muscle cell culture. Figure 6 shows the proliferation and mitochondrial activity of SMC on dHAM/PU composite membrane, with PU as the negative control and TCPS as the positive reference. During the 10-day culture interval, cells proliferated on all matrices, particularly rapidly increased at the first $7 \mathrm{~d}$ (OD values increased). There was insignificantly difference between OD values of dHAM/PU and of PU during the 10-day culture interval ( $p>0.05)$. However, OD value was higher significantly at day 3 than that at day 1 on both materials $\left({ }^{*} p<0.05\right.$ in Figure 6$)$. After then, the values on these two materials still increased with the time past. The general activities on both dHAM/PU and PU were lower than those of cells on TCPS, particularly after 7 or $10 \mathrm{~d}\left({ }^{\&} p<0.05\right.$, Figure 6). That is, cells proliferated on PU or dHAM/PU as the time went on, but they did not do as fast as those on TCPS becasue the cell adhension and proliferation properties of dHAM or PU surface are poorer than those of TCPS. It is reasonable since both dHAM's oversoftness and PU's 
hydrophobicity are disadvantageous for cells' attachment.

From H\&E staining, cells exhibited the spindle or triangular smooth muscle morphology (Figure 7).. The appearance or general density of cells on dHAM/PU increased during the culture time (Figure 7 a1-a4), just similar to the OD value tendency. At day 7, cells spread well and reached nearly confluence (Figure 7 a3). That's why the cell density (or OD value) increased slowly from day 7 to day 10 due to the cells' contact control. It is worth noting that cells on PU membrane displayed high growth and activities, which were lower insignificantly than those of cells on dHAM/PU. This is the motivation for us to select PU as the physical support to offer the mechanical strength while the biocompatibility from dHAM was maintained. The not bad cytocompatibility of PU material has repeatedly proved in our previous work [20-22].

The expression of smooth muscle $\alpha$-actin ( $\alpha$-SMA), a major constituent of the contractile apparatus of SMCs, was examined too. SMCs cultured on dHAM/PU had the higher $\alpha$-SMA fluorescence intensity with the longer culture time, suggesting the SMC phenotype and function were well maintained (Figure 8 a1-a4). Cells were able to reach confluence at day 10 (Figure 8 a4), which is of great importance once they are implanted in vivo. This tendency is coincident with the result of mitochondrial activity assay and morphology observation. Altogether, the combination of dHAM and PU was demonstrated to be a stable and straightforward treatment for implantable and cytocompatible mesh for clinic surgery purpose.

\subsection{In vivo testing}

On the basis of those in vitro cytocompatibility evaluations, dHAM/PU composite was implanted into uterine site through rabbits' abdomen with clinic PP mesh as the control, which would supply sufficient information for dHAM to be used as surgery mesh in future, The composite mesh was manually punched multi-poles in order to promote tissue infiltration and get the close appearance as PP mesh (Figure $9 \mathrm{a} 0$ ). The implanted rabbits were raised normally after the operation. The wound on the skin cured and the fur regenerated completely after one month post operation. Rabbits were sacrificed and examined at the $2^{\text {nd }}$ month. Viewing from eyesight, the implanted site was clear and no inflammation was obvious for both meshes, except for the adhesion of PP to the periphery viscera. H\&E staining of where mesh was originally implanted 
displayed that tissues were already infiltrated into the mesh pores (Figure 9 a and b). Tissues around dHAM/PU mesh indicated clear configuration (Figure $9 \mathrm{~b} 1$ ); the regenerated tissue integrated with the material while PU started to degrade due to fresh tissue invasion (Figure 9 b2, arrow), which is apparent in comparison of the appearance of PU before implantation (Figure 5a, inserted). The tissue enclosing PU was supposed to have regenerated freshly after implantation because there existed cell nuclei (b2, blue purple). The dHAM (without cell nuclei) must have been degraded and disappeared.

Whilst, tissues around PP fibers exhibited a disordered arrangement; excessive nuclei in cells suggested inflammation still existed around the fiber circumference after implantation for 2 months (Figure 9 a, inserted), which was caused by hydrophobicity and bio-inertia of PP material. After 10 months, the regenerated tissue around PP fibers showed the similar tissue hierarchy as that at $2^{\text {nd }}$ month (Figure 10 a). Interestingly, at site of dHAM/PU implantation, tissues remodeled orderly and normally. Some pores generated from microtome procedure during HE staining suggested dHAM/PU has been degraded into fragments (Figure $10 \mathrm{~b} 1$, arrow). The tissue around the fragments (Figure $10 \mathrm{~b} 2$, inserted) arranged orderly and almost similar to the neighbouring tissue (Figure $10 \mathrm{~b} 2$ ). The degradation of $\mathrm{PU}$ had once been investigated in vitro and in vivo. Generally, PU degraded more quickly in vivo than in vitro. It degraded into peeling off at day 100 when implanted subcutaneously in rat, but degraded into fragments at day 80 when implanted as muscular scaffold at porcine esophagus [21,22]. Mechanical stress during food ingestion and digestive enzymes brought by food bolus from mouth or the occasional backflow of gastric fluids from stomach were the reasons to have accelerated PU degradation. We thus considered that it was reasonable for PU to have degraded at 10 month in rabbit abdomen.

\section{Conculsions}

In summary, we established a technique to decellularize HAM with surfactant combining with lipase and DNAase, where surfactant and lipase were used to denature cell membrane and DNAase was used to enzymolyze DNA. Biodegradable poly(ester urethane) (PU) was used as a physical support for soft HAM because of its good biocompatibility. Results of in vitro 
cytocompatibility tests demonstrated that dHAM/PU composite can support primary SMC to grow and differentiate, with high proliferation, mitochondrial activity and special protein expression ( $\alpha$-SMA) after SMCs were cultured on dHAM/PU for $10 \mathrm{~d}$. The results of 10-month implantation revealed that dHAM/PU possessed much better biocompatibility than PP did. All these results convinced us that $\mathrm{dHAM} / \mathrm{PU}$ was promising to be used as biological mesh in clinic surgery. A more comprehensive study with different animals (even human beings) and variety of implantation sites will be processed in near future.

\section{Acknowledgements}

The authors gratefully acknowledge the financial support from the National Science Foundation (81171476, 81471797) and Social Development Project of Ningbo (2013C50031), China. This work was also sponsored by K.C. Wang Magna/Education Fund of Ningbo University.

\section{References}

1. N.J. Smart, M. Marshall, I.R. Daniels Biological meshes: a review of their use in abdominal wall hernia repair. Surgeon 10 (2012) 159-171.

2. G. Peppas, I.D. Gkegkes, M.C. Makris, et al., Biological mesh in hernia repair, abdominal wall defects, and reconstruction and treatment of pelvic organ prolapse: a review of the clinical evidence. Am. Surg. 76 (2010) 1290-1299.

3. N. Detta, C. Errico, D. Dinucci, et al., Novel electrospun polyurethane/gelatin composite meshes for vascular grafts. J. Mate. Sci. Mater. Med. 21 (2010) 1761-1769.

4. S.S. Yoon, Y. Chen, A. Kambadakone, et al., Surgical placement of biologic mesh spacers prior to external beam radiation for retroperitoneal and pelvic tumors. Pract. Radiat. Oncol. 3 (2013) 199-208.

5. M.A. Alamein, S. Stephens, Q. Liu, et al., Mass production of nanofibrous extracellular matrix with controlled 3D morphology for large-scale soft tissue regeneration. Tissu. Eng. C 19 (2013) 458-472.

6. M.R. Langenbach, J. Schmidt, M. Lazika, et al., Urological symptoms after laparoscopic 
hernia repair. Reduction with a variant of polypropylenemesh. Urolog. A 42 (2003) 375-381.

7. T. Poghosyan, N. Veyrie, N. Corigliano, et al., Retromuscular mesh repair of midline incisional hernia with polyester standard mesh: monocentric experience of 261 consecutive patients with a 5-year follow-up. World. J. Surg. 36 (2012) 782-790.

8. D.F. Williams, Carcinogenicity of implantable materials: experimental and epidemiological evidence. Int. Urogynecol. J. 25 (2014) 577-580.

9. C.R. Deeken, K.M. Faucher, B.D. Matthews, A review of the composition, characteristics, and effectiveness of barrier mesh prostheses utilized for laparoscopic ventral hernia repair. Surg. Endosc. 26 (2012) 566-575.

10. A.L. Siegel, M. Kim, M. Goldstein, et al., High incidence of vaginal mesh extrusion using the intravaginal slingplasty sling. J Urol 174 (2005) 1308-1311.

11. S.A. Michel, R.R. Vogels , N.D. Bouvy, et al., Utilization of flax fibers for biomedical applications. J. Biomed. Mater. Res. B 102 (2014) 477-487.

12. A. Prudente, C.L. Riccetto , M.M. Simoes, et al., Impregnation of implantable polypropylene mesh with S-nitrosoglutathione-loaded poly(vinyl alcohol). Colloid. Surf. B 108 (2013) $178-184$.

13. Y. Hao, D.H. Ma, D.G. Hwang, et al., Identification of antiangiogenic and antiinflammatory proteins in human amniotic membrane. Cornea 19 (2000) 348-352.

14. W. Zeng, Y. Li, G. Zeng, et al., Transplantation with cultured stem cells derived from the human amniotic membrane for corneal alkali burns: an experimental study. Ann. Clin. Lab. Sci. 44 (2014) 73-81.

15. G. Huang, S. Ji, P. Luo, et al., Accelerated expansion of epidermal keratinocyte and improved dermal reconstruction achieved by engineered amniotic membrane. Cell Transplant 22 (2013) $1831-1844$.

16. S. Wilshaw, J. Kearney, J. Fisher, et al., Production of an Acellular Amniotic Membrane Matrix for Use in Tissue Engineering. Tissue Eng 12 (2006) 2117-2129.

17. M. Saghizadeh, M.A. Winkler, A.A. Kramerov, et al., A simple alkaline method for decellularizing humanamniotic membrane for cell culture. Plos One 8 (2013) e79632. 
18. G.B. de Melo, J.A. Gomes, M.A. da Gloria, et al., Morphological assessment of different amniotic membrane epithelial denuding techniques. Arq. Bras. Oftalmol. 70 (2007) 407-411.

19. M. Saghizadeh, M.A. Winkler, A.A. Kramerov, et al., A simple alkaline method for decellularizing human amniotic membrane for cell culture. PLoS ONE 8 (2013) e79632.

20. C.F. Gong, L. Hou, Y.B. Zhu, et al., In vitro constitution of esophageal muscle tissue with endo-cyclic and exo-longitudinal patterns. ACS Appl. Mater. Interfaces 5 (2013) 6549-6555.

21. J.J Lv, L Chen, Y.B. Zhu, et al., Promoting Epithelium Regeneration for Esophageal Tissue Engineering through Basement Membrane Reconstitution. ACS Appl. Mater. Interfaces 6 2014) 4954-64.

22. Z.S. Shen, C. Kang, J.J. Chen, et al., Surface modification of polyurethane towards promoting the ex vivo cytocompatibility and in vivo biocompatibility for hypopharyngeal tissue engineering. J. Biomater. Appl. 28 (2013) 607-16.

23. L. Tatai, T.G. Moore, R. Adhikari, et al., Thermoplastic biodegradable polyurethanes: The effect of chain extender structure on properties and in-vitro degradation. Biomaterials 28 (2007) 5407-5417.

24. M. Boffito, E. Bernardi , S. Sartori , et al., A mechanical characterization of polymer scaffolds and films at the macroscale and nanoscale. J Biomed Mater Res A. 103 (2015) 162-169.

25. M. Farazdaghi, Electron microscopy of human amniotic membrane. Advances in Tissue Banking, Vol. 5. New York: World Scientific Publishing Co. Ltd., 2001.

26. K.H. Ulrich, I.M. Marc, V.M. Jesper, The mechanism of detergent solubilization of liposomes and protein-containing membranes. Biophys. J. 75 (1998) 2932-2946.

27. A. Porzionato, M. Sfriso, V. Macchi, et al., Decellularized omentum as novel biologic scaffold for reconstructive surgery and regenerative medicine. Euro. J. Histochem. 57 (2013) e4.

28. Y.B. Zhu, C.Y. Gao, T. He, et al., Endothelium regeneration on luminal surface of polyurethane vascular scaffold modified with diamine and covalently grafted with gelatin. Biomaterials 25 (2004) 423-430. 


\section{Captions:}

Figure 1 H\&E staining of HAM after decellularization with methods of SD, HD, and LD. (a0) the original HAM (cross section), arrow shows the amniotic epithelium and triangle indicates connective tissue. The inserted is the topology of amniotic membrane stained by $\mathrm{H} \& \mathrm{E}$ with nucleus displayed as purple particles. (a1) Treated with SD method: 1\% Triton X-100 for $1 \mathrm{~d}$ and DNAase (2000 U/L) for $3 \mathrm{~h}$ at $37^{\circ} \mathrm{C}$; (a2) Treated with HD method: $\mathrm{NaCl}$ solution (3M) for $1 \mathrm{~d}$ and DNAase (2000 U/L) for $3 \mathrm{~h}$ at $37^{\circ} \mathrm{C}$. (a3) Treated with LD method: lipase/PBS (2000 U/L) for $10 \mathrm{~h}$ and DNAase $(2000 \mathrm{U} / \mathrm{L})$ for $3 \mathrm{~h}$ at $37^{\circ} \mathrm{C}$. The inserted is the HAM treated by lipase/PBS (1000 $\mathrm{U} / \mathrm{L})$ and DNAase. Scale bar, $20 \mu \mathrm{m}$.

Figure 2 Nuclei exhibitions after DAPI staining (membrane topography) of HAM decellularized with methods of SD, HD, and LD. (a0) Original HAM. (a1) (a3) the same treatment as Figure 1 description. Scale bar, $100 \mu \mathrm{m}$.

Figure 3 H\&E (a1, cross-section) and DAPI staining (a2) of HAM treated with SLD method: 1\% Triton X-100 for $14 \mathrm{~h}+2000 \mathrm{U} / \mathrm{L}$ lipase solution for $10 \mathrm{~h}+2000 \mathrm{U} / \mathrm{L}$ DNAase/PBS for $3 \mathrm{~h}$ at $37^{\circ} \mathrm{C}$.

Figure 4 Topography of HAM (a) and DHAM (b) treated with SLD method, observed under SEM. Figure $5 \mathrm{H} \& \mathrm{E}$ staining of dHAM/PU (a, the inserted is magnified and arrow pointed the interface) and SEM observation (b) of dHAM/PU composite. The upper layer is dHAM and the underline is $\mathrm{PU}$ in (b). The inserted is a magnified picture.

Figure 6 Mitochondrial activity assay. Cells were seeded at the density of $4.0 \times 10^{4} / \mathrm{mL}$ and cultured for 10d. $p>0.05$ for OD value difference between PU and dHAM/PU, but $p<0.05$ between day 1 and day 3 on both materials $\left(^{*}\right)$, and between materials and TCPS after culture for 7 or $10 \mathrm{~d}\left({ }^{\&}\right)$.

Figure 7 Cell morphology after cells were stained with H\&E dye. SMCs were seeded on dHAM/PU at the density of $4.0 \times 10^{4} / \mathrm{mL}$ and cultured for $1 \mathrm{~d}(\mathrm{a} 1), 3 \mathrm{~d}(\mathrm{a} 2), 7 \mathrm{~d}(\mathrm{a} 3)$ and $10 \mathrm{~d}(\mathrm{a} 4)$. Scale bar, $100 \mu \mathrm{m}$.

Figure 8 Immunofluorescent staining of SMCs. Cells were cultured on dHAM/PU for 1d (a1), $3 \mathrm{~d}$

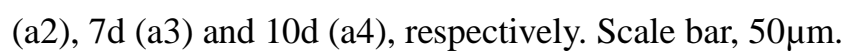


Figure 9 H\&E staining of PP (a) and dHAM/PU mesh (b) implanted at uterine site for 2 months. b2 is magnified from b1. (a0) Overview of implanting operation, red arrow shows PP (upper inserted) and black indicates dHAM/PU mesh (lower inserted). The inserted displayed the appearance of clinic PP and dHAM/PU mesh. Arrow in (a) pointed PP fiber, and the inserted is a magnified picture showing the tissue microscopy around PP fiber. Scale bar in (a) and (b1) is $200 \mu \mathrm{m}$ and in (b2) is $20 \mu \mathrm{m}$.

Figure $10 \mathrm{H} \& \mathrm{E}$ staining of PP (a) and dHAM/PU mesh (b) after implanted for 10 months. b2

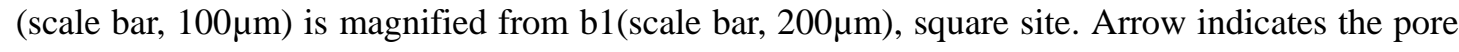
originated from PU fragment. The inserted in b2 showed the tissue around PU fragment with higher magnification. 


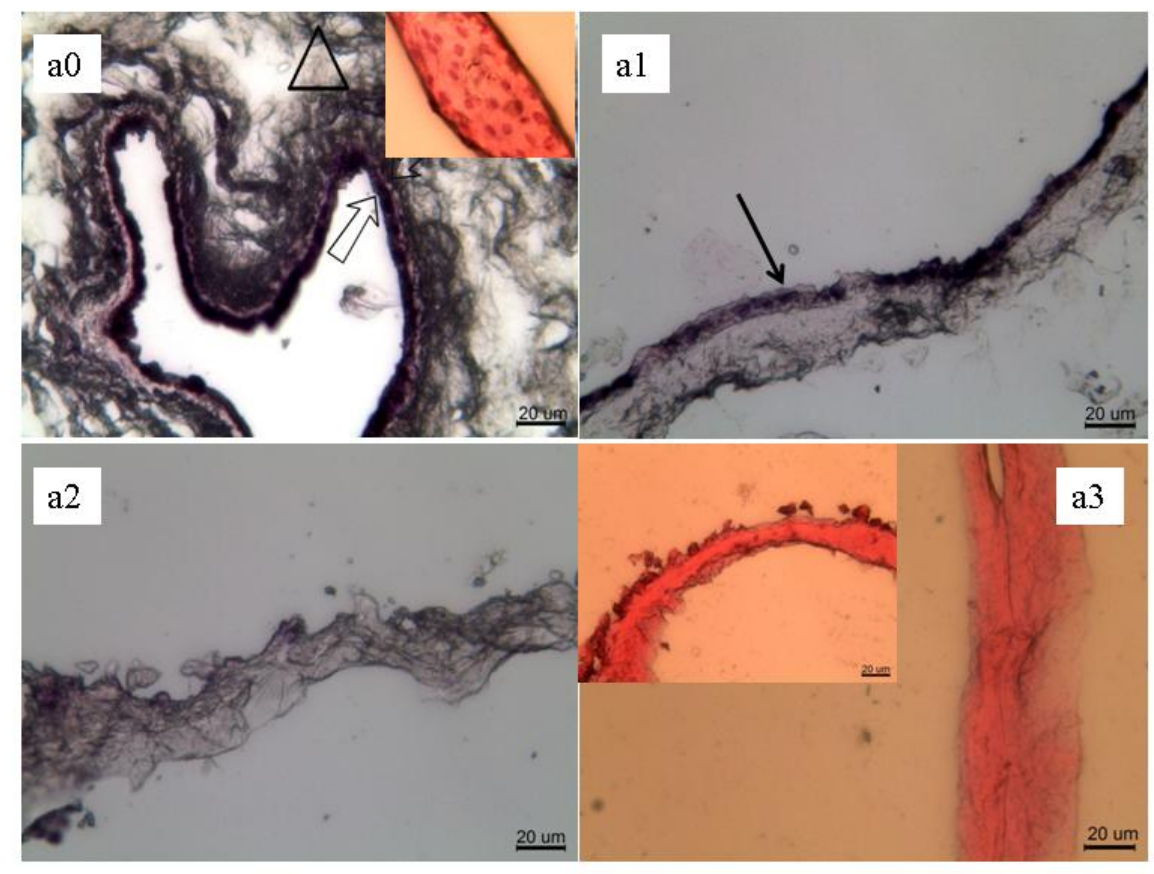

Figure 1 


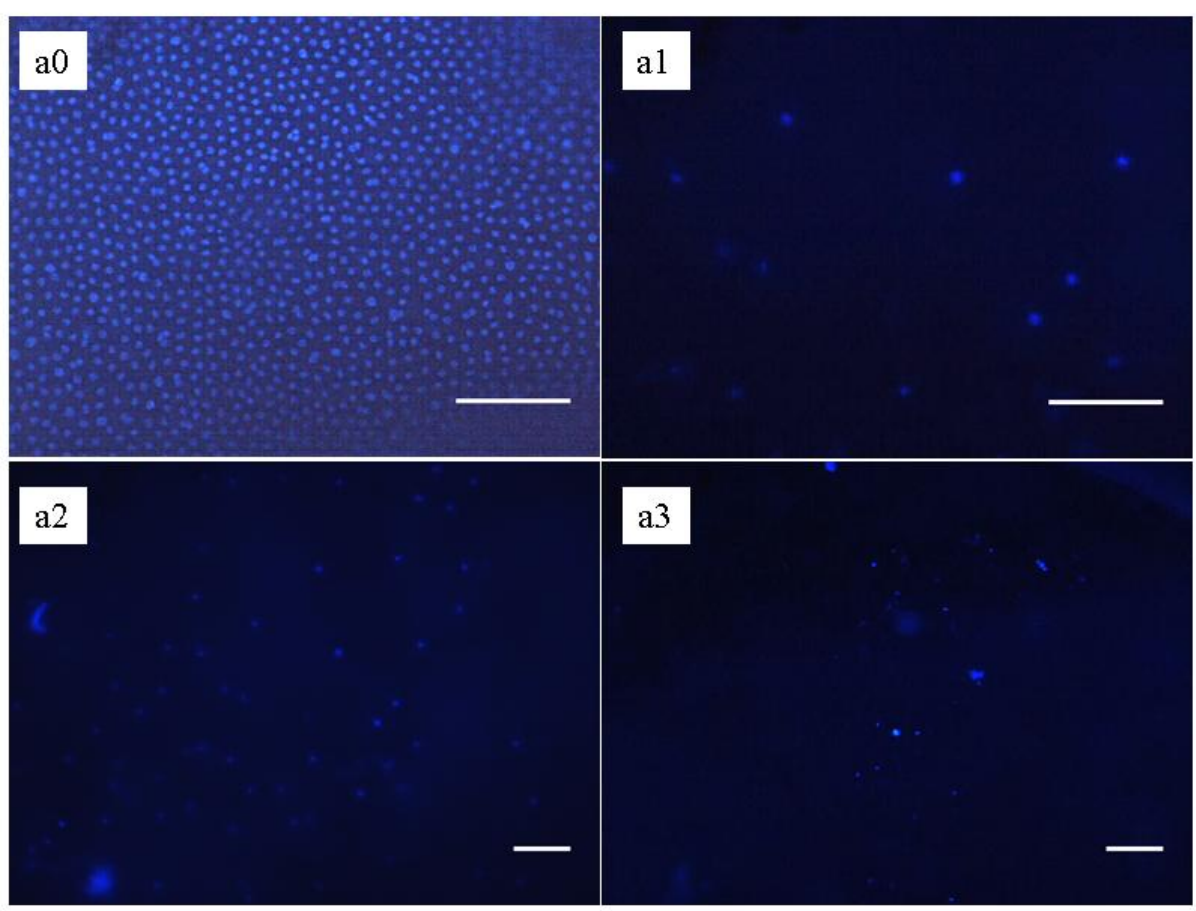

Figure 2 


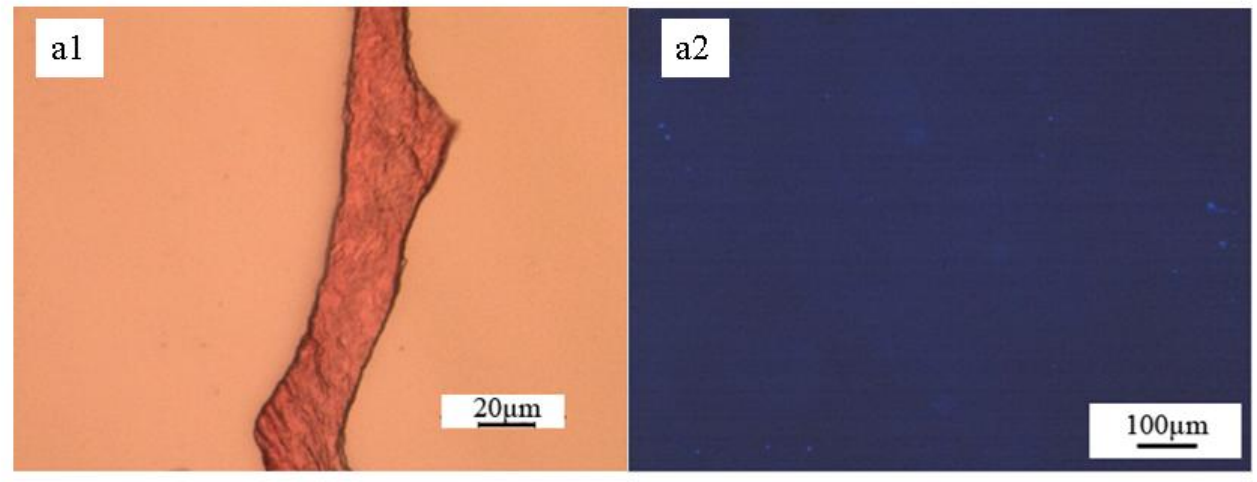

Figure 3 


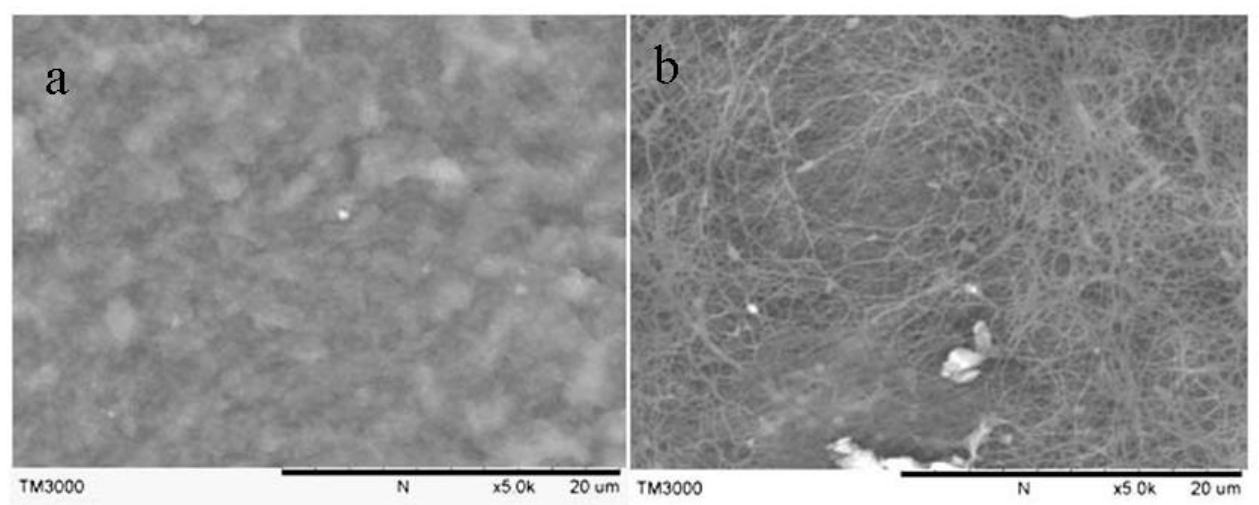

Figure 4 


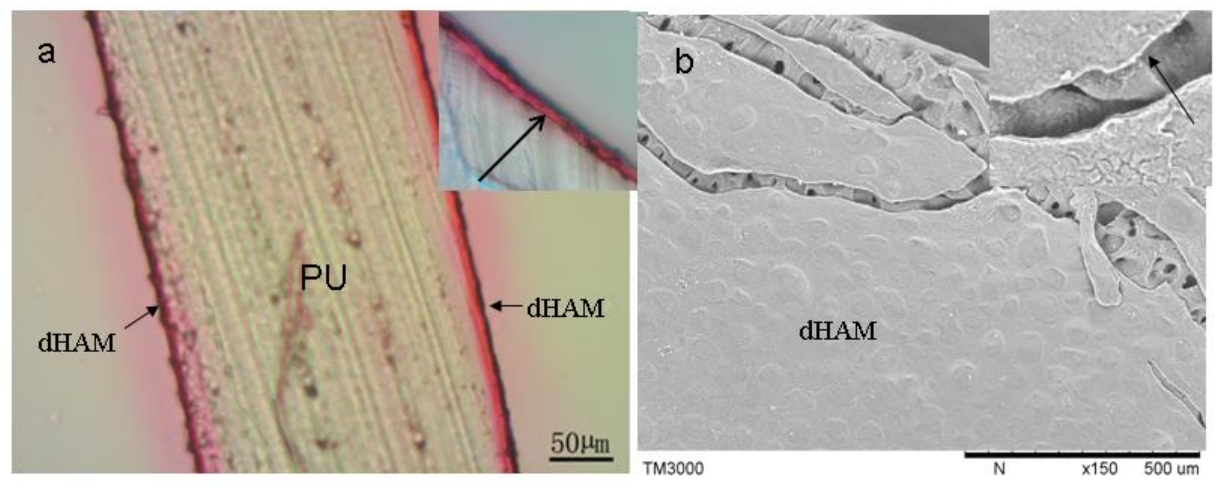

Figure 5 


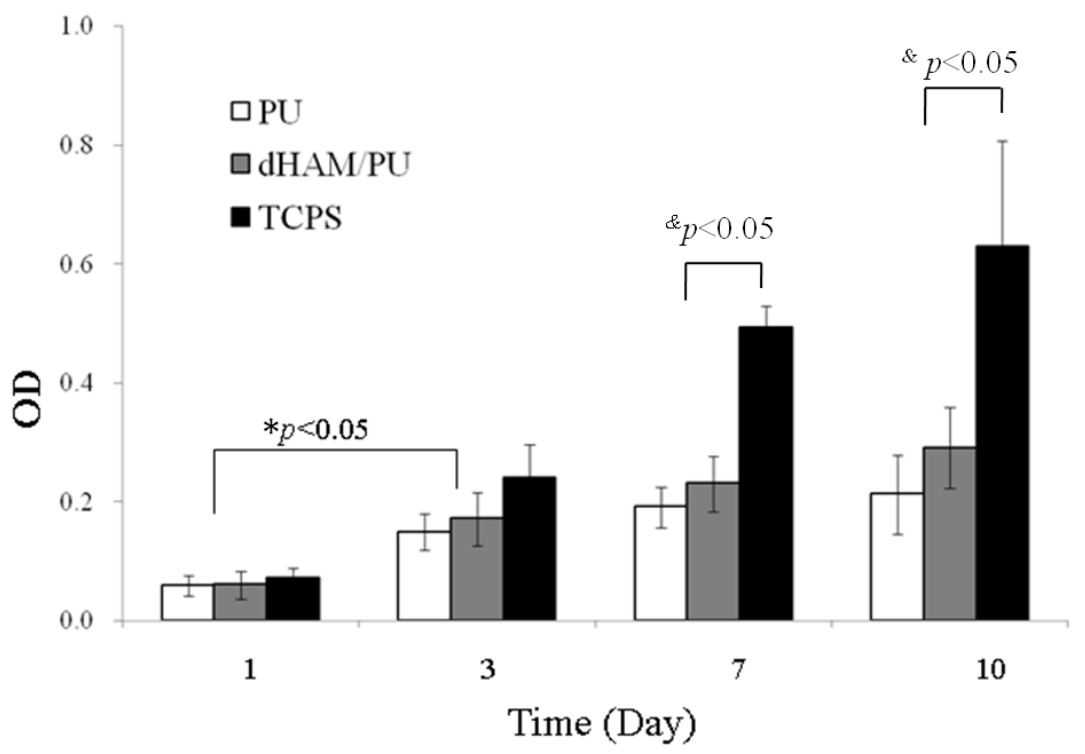

Figure 6 


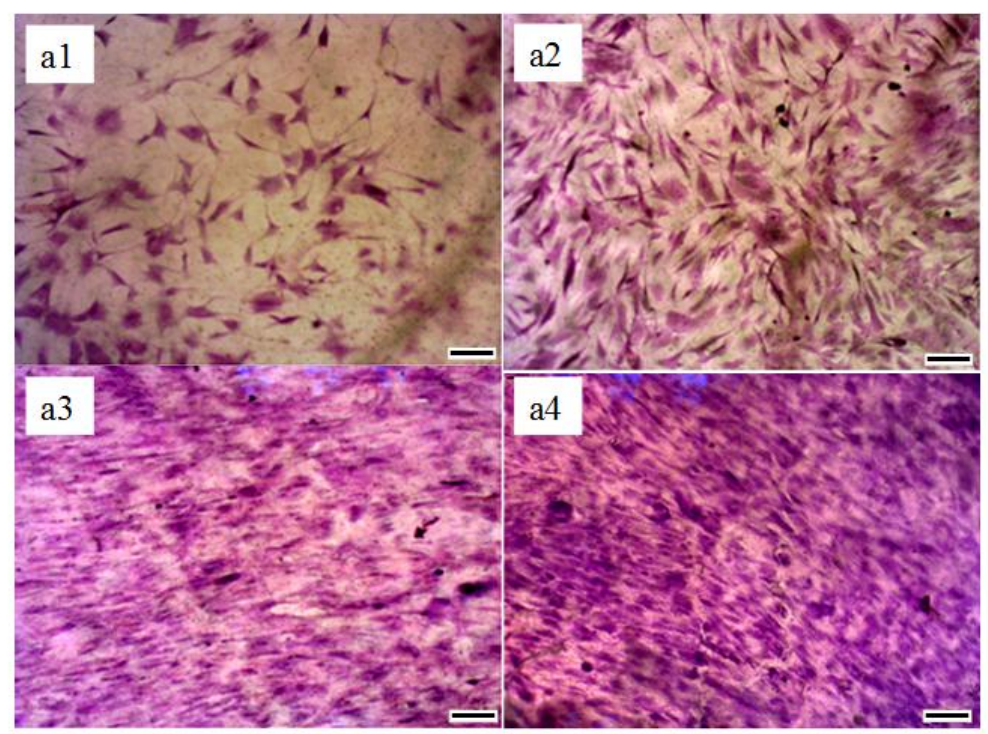

Figure 7 


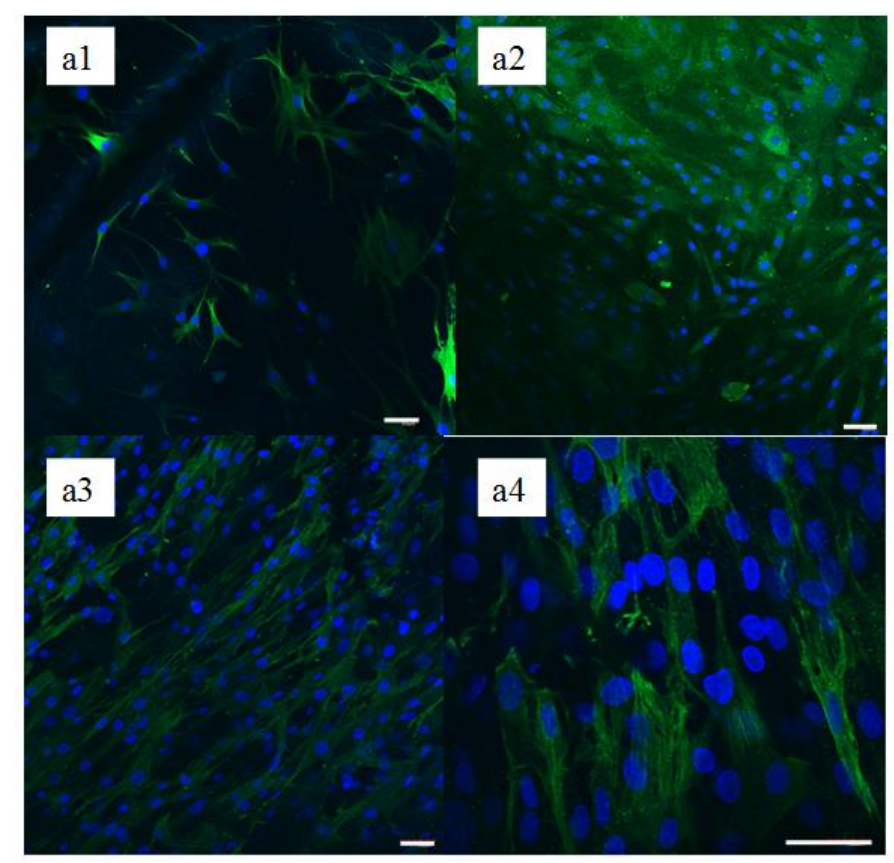

Figure 8 


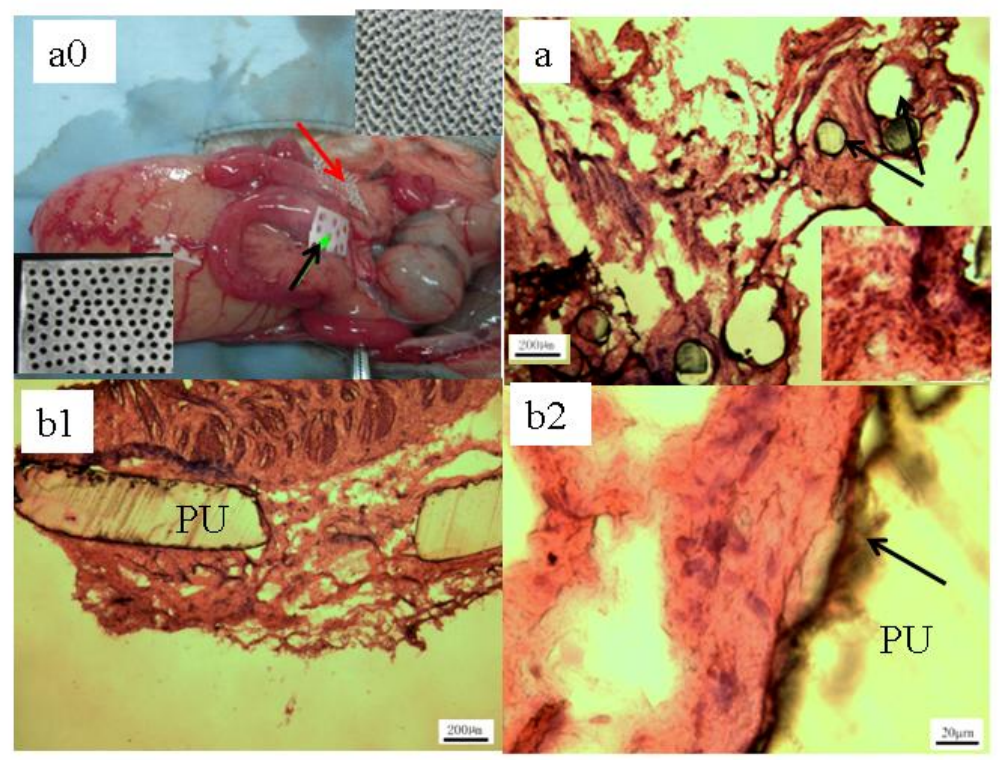

Figure 9 


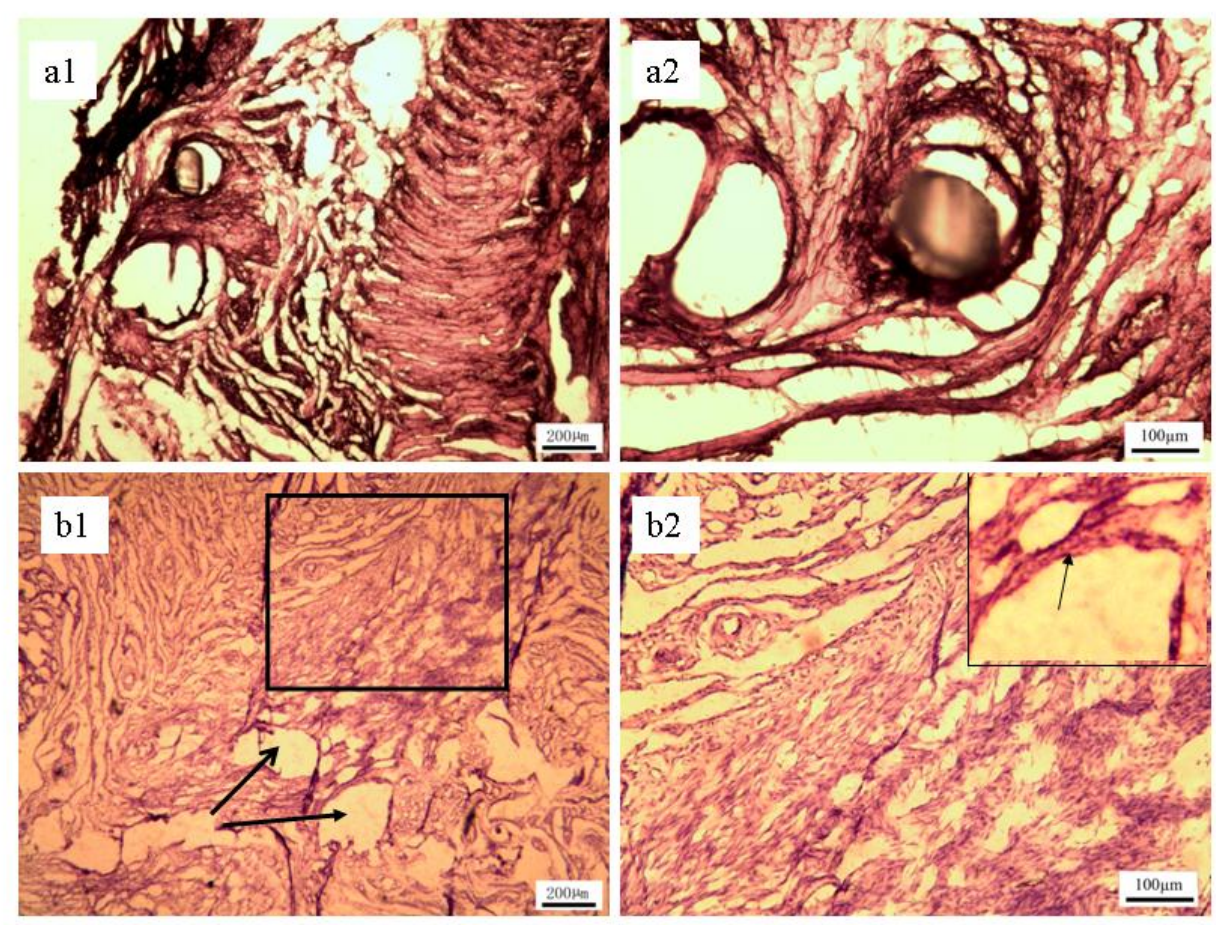

Figure 10 
Graphical Abstract:

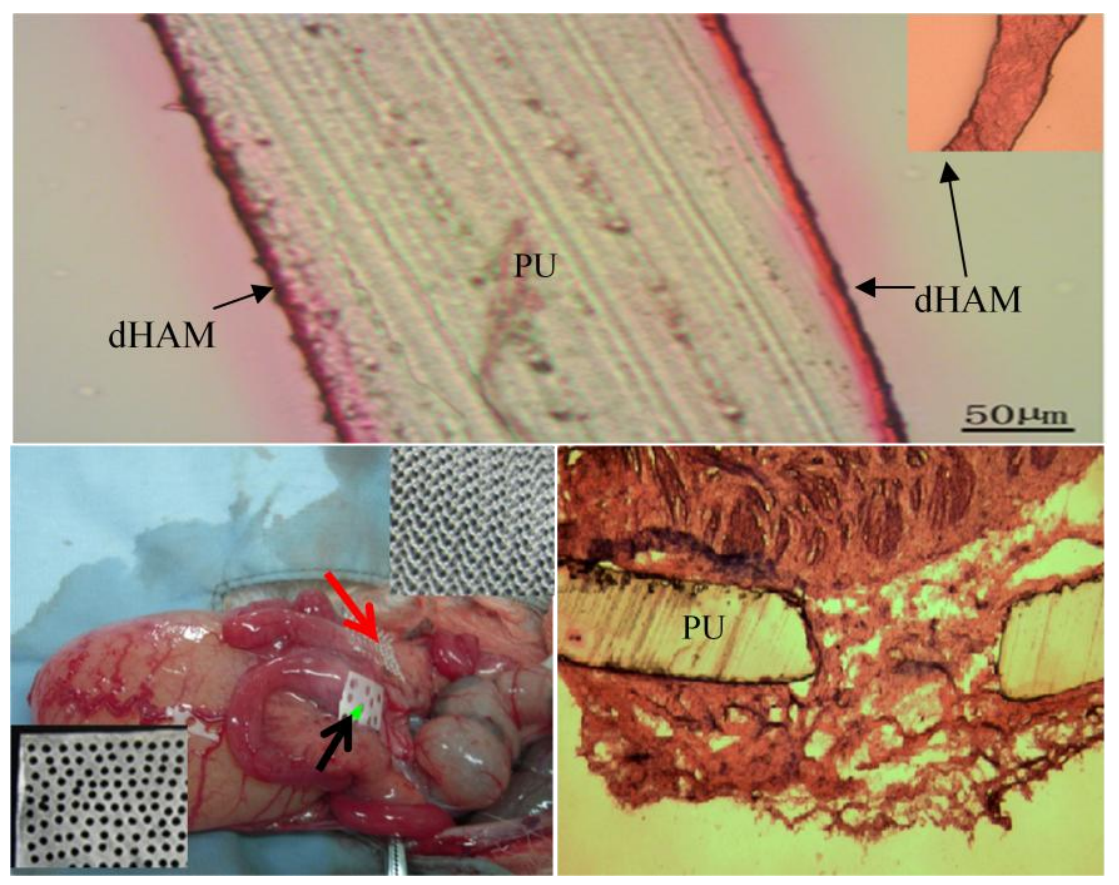

